\title{
A digital data acquisition system optimized for spectrometry with liquid scintillation detectors
}

\section{Marocco}

Associazione Euratom-ENEA sulla Fusione

C.P. 65, Frascati I-00044, Roma (Italy)

E-mail: marocco@.frascati.enea.it

\section{Riva, B. Esposito, L. Bertalot}

Associazione Euratom-ENEA sulla Fusione

C.P. 65, Frascati I-00044, Roma (Italy)

\section{A. Zimbal ${ }^{1}$}

Physikalisch-Technische Bundesanstalt

Bundesallee 100, D-38116, Braunschweig (Germany)

\begin{abstract}
The prototype of a new concept FPGA-based digital acquisition board has been developed for application with scintillation detectors. The board performs digitisation of pulses at 200 MSamples/s with 14-bit resolution. A Dynamic Windows Data Acquisition (DWDA) method is used such that data sampling occurs only when pulses are present and for a time window depending on the width of each pulse. This allows to reduce the amount of stored data and, at the same time, to handle count rates up to the $\mathrm{MHz}$ range. Peak identification, baseline correction, pile-up removal, neutron $/ \gamma$ ray separation and pulse height analysis are performed by means of a LabVIEW ${ }^{\mathrm{TM}}$ program. We show the results of measurements carried out with an NE213 liquid scintillation detector at the PTB (Physikalisch-Technische Bundesanstalt) accelerator facility in Braunschweig (Germany) both with $2.5 \mathrm{MeV}$ and $14 \mathrm{MeV}$ neutrons. The measurements have been performed at different source to detector distances as well as various detection angles. In the digital branch of the acquisition, the pulses have been stored on disk and analysed after the experiment. The comparison between the PTB standard analogue pulse processing system and the new digital system is presented and discussed, in particular in terms of energy resolution, dependence of the pulse height spectra on different total count rates, system stability with time, pile-up rejection and neutron $/ \gamma$-ray separation capability.
\end{abstract}

International Workshop on Fast Neutron Detectors

University of Cape Town, South Africa

April 3-6, 2006

\footnotetext{
${ }^{1}$ Speaker 


\section{Introduction}

Recent developments in digital techniques have led to the use of fast digitizers for sampling fast pulses from scintillators with $\mathrm{n} / \gamma$ discrimination properties (such as NE213, BC501A and stilbene) so that the $\mathrm{n} / \gamma$ separation is simply performed by software means: this technique is called digital pulse shape discrimination (DPSD) [1]. A prototype 14-bit 200 MSamples/s digital system has been developed in ENEA-Frascati: its main features are the capability of acquiring data continuously for any preset time interval and the possibility to perform on-line elaboration prior to data storage (Section 2). This system, together with a standard analogue system, has been used for neutron spectrometry measurements carried out with an NE213 scintillator at the PTB (Physikalisch-Technische Bundesanstalt, Braunschweig, Germany) accelerator facility with monoenergetic neutrons from the reactions $d+d \rightarrow{ }^{3} \mathrm{He}+n$ and $\mathrm{d}+\mathrm{t} \rightarrow \alpha+\mathrm{n}$ (Section 3). The results of the comparison of the performances of the two systems are reported and discussed (Sections 4 and 5).

\section{The digital system}

The prototype 14-bit 200 MSamples/s digital system consists of a high speed PCI board (National Instrument 6534), two 14-bit 100 MSamples/s ADCs (Analog Devices) coupled in interleaved mode (in order to obtain an actual sampling rate of $200 \mathrm{MSamples} / \mathrm{s}$ ), an Altera 1S25 field programmable gated array (FPGA) and a LabVIEW ${ }^{\mathrm{TM}}$ software devoted to acquisition handling and pulse analysis.

The analogue signal from the photomultiplier (PMT) is digitized in discrete bunches of data of variable number of samples (windows). A pre-programmed number of samples (minimum window length) is acquired when the signal crosses a pre-set hardware threshold: however, the length of the time window is automatically increased to account for long pulses or in case further pulses arrive. The system associates to each window a length value (number of samples) and a time stamp value corresponding to the time of the first hardware threshold crossing. This Dynamic Windows Data Acquisition (DWDA) logic allows acquiring only the data that actually carry information leading to a strong data compression that is of great help for the successive data transfer/storage [2]. The digitized data are sent to a PC through a PCI bus and first stored in a selectable portion of the RAM. When the allocated RAM is full the acquisition stops and the data are saved to disk. The maximum count rate that can be handled by the system without losses (until the RAM is full) is determined by the maximum transfer rate of the digital PCI board $(80 \mathrm{MB} / \mathrm{s})$ and the typical pulse length.

The configuration of the board parameters is handled by a set of $\mathrm{C}++$ routines that also perform a first data elaboration in which pulse, length and time stamp data are separated and prepared for successive analysis. These routines are integrated in a wider LabVIEW ${ }^{\mathrm{TM}}$ environment that manages the $n / \gamma$ separation, pile-up rejection, $n / \gamma$ pulse height spectrum and count rate evaluation. The first elaboration stage is the "baseline evaluation" in which it is possible, for each window, to subtract the baseline using the average of a selectable number of 
samples taken at the beginning and/or at the end of the window. In the subsequent step a "peak detection" algorithm recognises the number of pulses in each window above a selectable software threshold: if more than one pulse is found the window is labelled as pile-up, and pulses in such a window are considered just for count rate purposes. Single pulse windows instead flow trough an "integration" stage in which the pulse is integrated in three different time windows: full window for energy evaluation, short and long windows for $n / \gamma$ discrimination [3]. The integral information is then used in the "pulse height analysis" (PHA) stage to produce separate $n$ and $\gamma$ pulse height spectra. A "count rate" stage finally merges all previous information to give total count rate, single pulses count rate, pile-up count rate and separate $n$ and $\gamma$ count rates.

The PTB detector uses a light emitting diode (LED) to correct for gain variations of the PMT produced by variation of the count rate [4]. An "LED recognition" stage has therefore been added to the analysis software in which single LED pulses are separated from the others and integrated, in order to be later on used for correction of single pulse integrals according to the following procedure: an average value of the LED integral is evaluated every $\Delta t$ seconds $\left(\left\langle\left(L E D_{\text {neutron measurement }}\right)\right\rangle_{\Delta t}\right)$; the full integrals of all pulses occurring within $\Delta t$ are corrected by the ratio $L E D_{\text {reference }} /\left\langle\left(L E D_{\text {neutron measurement }}\right)\right\rangle_{\Delta t}$, where $L E D_{\text {reference }}$ is a reference LED integral corresponding to the maximum of the Gaussian distribution of LED integrals of a $\gamma$ calibration (low count rate).

\section{Experiment set-up}

The aim of the experiment was to benchmark the ENEA digital system using a welldefined source of monoenergetic neutrons and to evaluate its performances against a fully characterised analogue system used for metrological applications. An NE213 liquid scintillator characterized at PTB [4] has been exposed to the neutron beam produced by the PTB accelerator and the PMT signals have been acquired both with the standard PTB analogue system and with ENEA digital system; it has been chosen to acquire data from each system separately in subsequent irradiations performed in the same conditions. In the digital case the direct signal from the PMT anode has been used without any amplification stage. In the analogue case, the signal from the $9^{\text {th }}$ dynode of the PMT was integrated with a charge sensitive preamplifier and a main amplifier (delay line amplifier Ortec 460 with a shaping time of $1 \mu \mathrm{s}$ ). The signals from the main amplifier are used for PHA with conventional NIM-ADCs (Silena 7423 UHS). The fast signal from the anode has been used for $n / \gamma$ separation performed by a Canberra 2160 pulse shape discrimination module.

Detector irradiations have been performed both with $2.5 \mathrm{MeV}$ (DD) and $14 \mathrm{MeV}$ (DT) neutrons, at different source to detector distances as well as at various detection angles. Acquisitions using a ${ }^{207} \mathrm{Bi}$ gamma source have also been performed at different times during the experiment for energy calibration and stability check purposes. The maximum available RAM of $\sim 0.7 \mathrm{~GB}$ [2] has limited the time duration and therefore the counting statistics in the digital acquisitions. 
Since the PMT signals are negative while the input range of the digital system is $\pm 1.1 \mathrm{~V}$, only half of the total input range of the ADC's was used. Moreover, the standard working conditions of the PTB system have been mainly used throughout the experiment and this led in particular to the use of a PMT high voltage such that the pulse amplitude range normally did not fully match the ADC negative input range. Due to these operating conditions the actual bit resolution of the digital system was slightly less than 13 bit.

Given the experimental conditions, both hardware and software parameters of the DPSD system were carefully set up in order to obtain the best performances. Considering the noise level of the PMT signal the hardware ADC threshold was set to $\sim-18 \mathrm{mV}$; taking into account the typical time duration of the expected largest amplitude pulses (for $14 \mathrm{MeV}$ neutrons), a minimum window length of 96 samples ( $480 \mathrm{~ns}$ ) has been chosen in order to have as much as possible information on each single pulse for a detailed analysis (e.g.: baseline correction, integration parameters, etc.): in these particular conditions a continuous count rate of $\sim 350 \mathrm{kHz}$ can be sustained. On the software side four parameters mainly affect the DPSD final output: number and position of the baseline samples, full, short and long window lengths (see Section 2). Their best values have been determined off-line after data acquisition: only pre-pulse samples were used for the baseline determination (typically 8, corresponding to $40 \mathrm{~ns}$ ); the length of the full window was determined for each pulse through an algorithm comparing the pre-pulse baseline value with a moving average baseline value evaluated starting from the peak of the pulse towards the end of the window; the short and long windows were set, respectively, to 5 samples $(25 \mathrm{~ns})$ and 24 samples (120 ns) starting at the peak of the pulse. Following this analysis, it is found that, in this experimental set-up, the necessary minimum window length is roughly 22 samples; as the average DD and DT pulse lengths are, respectively, 33 and 63 samples, this translates into sustainable continuous count rates of about $900 \mathrm{kHz}$ (DD) and 540 $\mathrm{kHz}(\mathrm{DT})$.

\section{Results}

The stability of the digital system was evaluated comparing the pulse height spectra (PHS) of different gamma calibrations taken during the experiment. In these low count rate acquisitions the LED signals are stable in position, except for small shifts due to drifts of the DPSD electronics. The differences in the peak position of the LED gaussian distributions for the digital system were found to be at most $0.7 \%$. After normalization to the same position of the LED in the spectrum, the position of the Compton edges of the various calibrations were found to be identical (with maximum differences below $0.5 \%$ ). This allowed to select a single gamma calibration and to use it as energy reference for all the neutron acquisitions. The energy calibration was performed with the same procedure for the analogue and digital data using the Compton edges of three lines of the ${ }^{207} \mathrm{Bi}$ source. A comparison between two energy calibrated ${ }^{207} \mathrm{Bi}$ spectra obtained with the analogue and digital system is reported in Fig.1 (all PHS shown in this paper are expressed in terms of the electron-equivalent energy $\left.E_{\mathrm{ee}}\right)$. 
A digital data acquisition system optimized for spectrometry with liquid scintillation detectors

The two spectra overlap, the only difference being a slight broadening of the ${ }^{207} \mathrm{Bi}$ lines in the digital PHS, indicating a slightly lower pulse height resolution. Similarly, the two LED peaks have the same energy (as determined with a ${ }^{207} \mathrm{Bi}$ source) while the FWHM of the LED appears to be slightly broader in the digital case. The calibration of the recorded PHS in terms of electron energies has been made using calculated Compton distributions for the three different $\gamma$-lines of a ${ }^{207} \mathrm{Bi}$ source, varying in both systems the ADC offset and gain factor, and using a broadening of the calculated distributions according to the relation $d L / L=\left(A^{2}+B^{2} / L+C^{2} / L^{2}\right)^{1 / 2}$ [5] with $d L / L=$ pulse height resolution for the light output $L$ and the resolution parameters $A, B, C$.
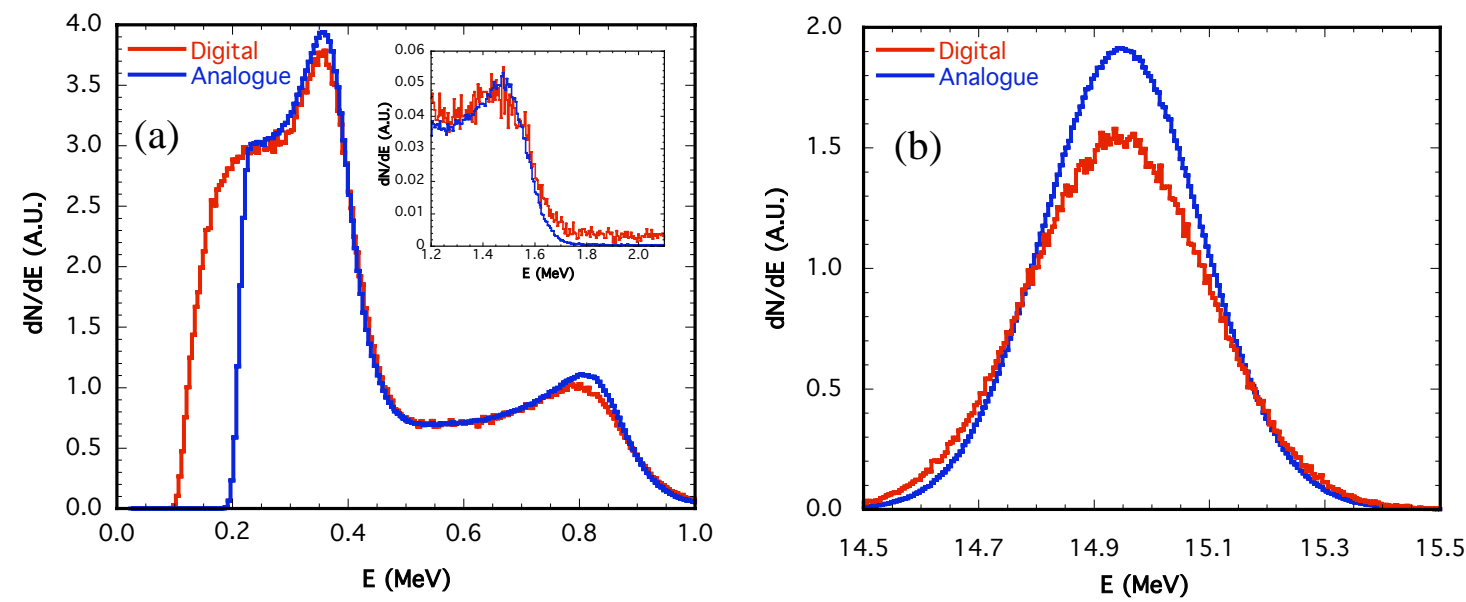

Figure 1: Digital and analogue PHS (energy calibrated using a ${ }^{207} \mathrm{Bi}$ source: both spectra have been normalized to the total integral between $200 \mathrm{keV}$ and $2 \mathrm{MeV}$ ) (a); Digital and analogue PHS showing the gaussian distribution of the LED (the energy scale was determined using a ${ }^{207} \mathrm{Bi}$ source) (b).
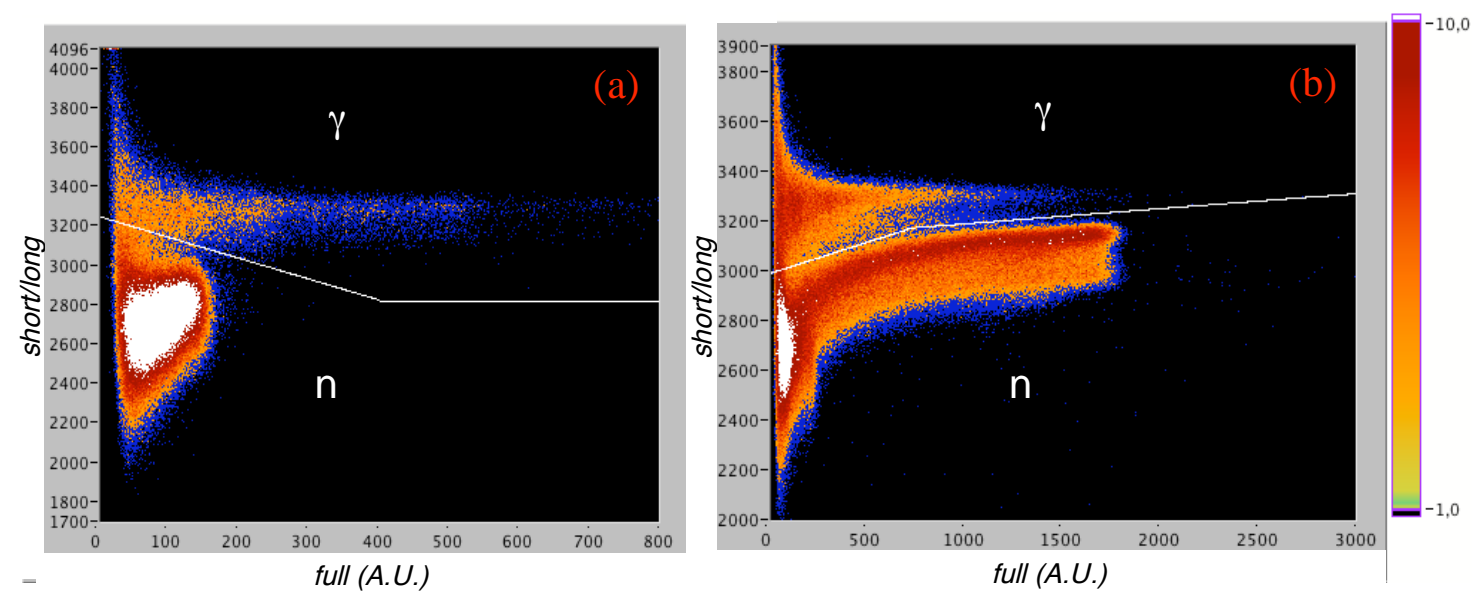

Figure 2: $\mathrm{n} / \gamma$ separation plots for a DD $\left(106^{\circ}, 7.5 \mathrm{kHz}\right.$ total count rate $)$ acquisition (a) and for a DT $\left(0^{\circ}, 13 \mathrm{kHz}\right.$ total count rate) acquisition (b).

The best agreement was reached by changing the parameter $B$, describing the statistics of the production and multiplication of the photoelectrons, from $B_{\text {analogue }}=9.31$ to $B_{\text {digital }}=12.13$. The other parameters are found to be the same, but measurements with other gamma and neutron energies are needed to confirm this. These results give indications that the differences found in 
the pulse height resolution might be explained as an effect due to different statistics in the multiplication of electrons in the last stages of the PMT, producing different signals at the PMT anode (digital case) with respect to the $9^{\text {th }}$ dynode (analogue case); however further investigations are needed to clarify this point.

Digital neutron data have been corrected for PMT gain variations and then energy calibrated using the same reference ${ }^{207} \mathrm{Bi}$ gamma acquisition. The gain correction has been performed using the procedure described in Section 2, using an averaging time interval $\Delta t=100$ $\mathrm{ms}$ (no spectrum variations are found using lower $\Delta t$ down to $1 \mathrm{~ms}$ ).

Fig.2 shows typical $n / \gamma$ separation plots for DD and DT irradiations: normalized values of the full (energy) integral and the ratio short/long integrals are reported respectively on the $\mathrm{x}$ and $y$ axes; the colour scale is proportional to the number of counts; $n / \gamma$ separation lines are also shown (white lines).

The comparison between the analogue and digital PHS in DD $\left(106^{\circ}\right)$ and DT $\left(98^{\circ}\right)$ irradiations are shown in Fig.3. The energy scale is in agreement at $2.5 \mathrm{MeV}$ while a down-shift of about $1.4 \%$ is observed at the proton recoil edge of the $14 \mathrm{MeV}$ neutron spectrum in the digital case: this effect may be due to non-linearity introduced by the last amplification stages of the PMT. As in the $\gamma$ calibration PHS the pulse height resolution is slightly lower in the digital cases.
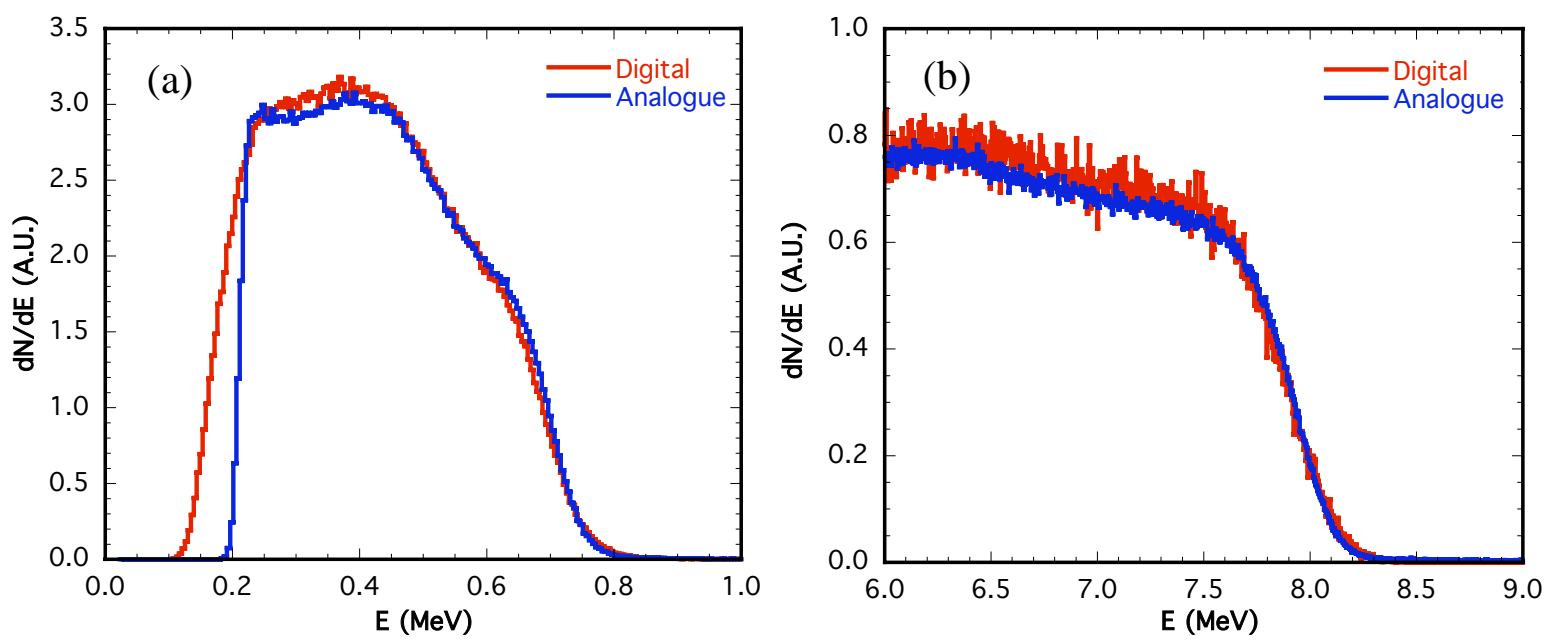

Figure 3: Digital and analogue PHS: (a) DD irradiation $\left(106^{\circ}, \sim 7.5 \mathrm{kHz}\right.$ total count rate, both spectra normalized to the total integral between $300 \mathrm{keV}$ and $1 \mathrm{MeV})$; (b) DT irradiation $\left(98^{\circ}, \sim 13 \mathrm{kHz}\right.$ total count rate, spectra normalized to the total integral between $6 \mathrm{MeV}$ and $9 \mathrm{MeV}$ ); the energy scale of the digital spectrum has been multiplied by a factor 1.014. Noise in the digital spectra is due to lower counting statistics (see above, Section 3).

A set of irradiations at low source to detector distance has been performed to test the capability of the digital system to handle high total count rates. While the analogue system is limited to about $30 \mathrm{kHz}$ (for spectrometry without significant distortion of the PHS with pileup), in the digital case over $200 \mathrm{kHz}$ has been measured (Fig.4): the count rates shown have been corrected for the pile-up assuming that the $n, \gamma$ and LED distribution in pile-up events is the same as in single events. The corresponding PHS is still close to the PHS measured at lower 
count rates, and only a small deviation $(\sim 1 \%)$ in the position of the proton recoil edge of the 14 $\mathrm{MeV}$ neutrons, which may be due to PMT saturation effects, is observed (Fig.5).
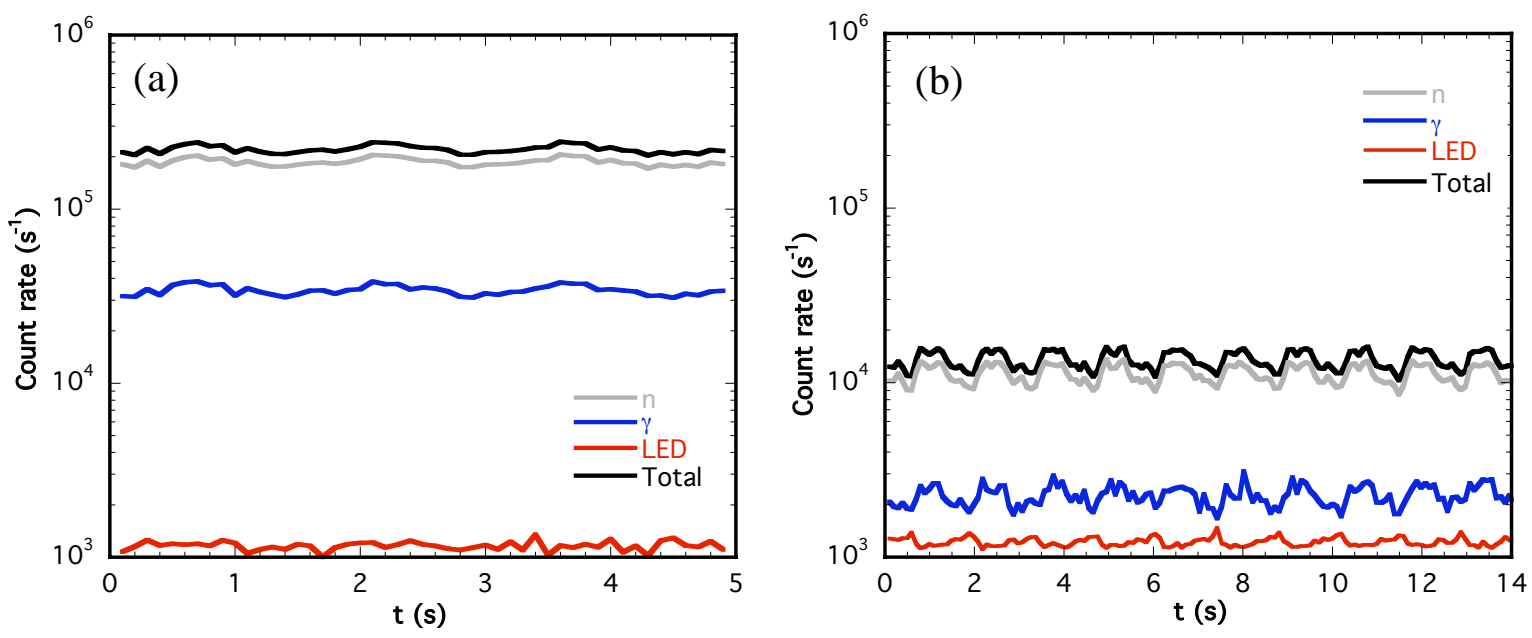

Figure 4: Different count rates from the digital system for two DT, $0^{\circ}$ irradiations: (a) $\sim 240 \mathrm{kHz}$ total count rate; (b) $\sim 13 \mathrm{kHz}$ total count rate. Notice in (b) the $\sim 1 \mathrm{~Hz}$ modulation in the count rates due to the rotation of the neutronproducing target.
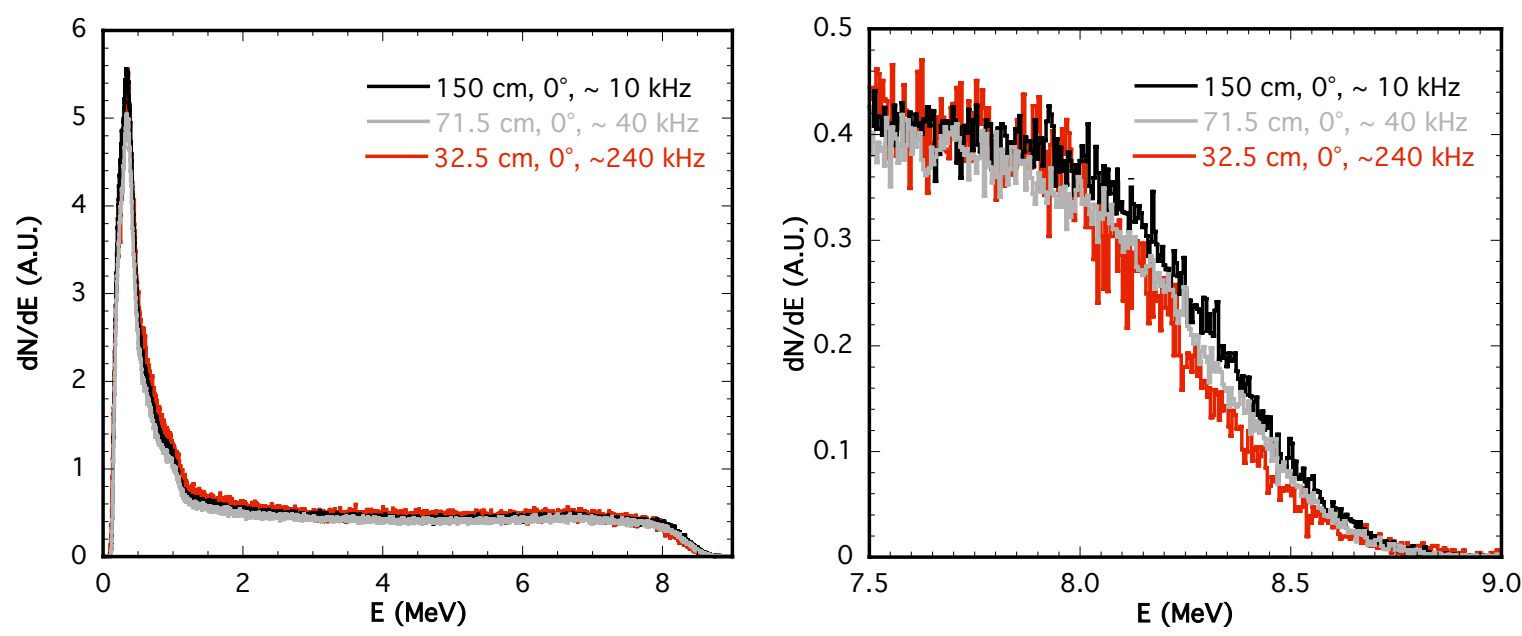

Figure 5: Digital DT spectra $\left(0^{\circ}\right)$ at different source to detector distances.

\section{Conclusions}

The first results of the comparison between the analogue system used at PTB for neutron spectroscopy applications with NE213 scintillators and a prototype digital system developed in ENEA indicate that: 1) the PHS are in close agreement (the observed slight difference of the pulse height resolution and $1.4 \%$ shift of the observed energy of $14 \mathrm{MeV}$ recoil protons being probably due to the use of the anode for the digital system instead of the $9^{\text {th }}$ dynode for the analogue system);2) the digital system has shown the possibility of measuring simultaneously PHS of $2.5 \mathrm{MeV}$ and $14 \mathrm{MeV}$ neutrons up to total count rates of $240 \mathrm{kHz}$, with spectral 
A digital data acquisition system optimized for spectrometry with liquid scintillation detectors

distortion within $1 \%$. Evaluation of the dependence of these effects on the PMT high voltage or using other set-ups such as very fast preamplifiers with the anode or dynode signals is in progress. Moreover, the $\mathrm{n} / \gamma$ separation must still be optimized by varying the integration times and checking the resulting figures of merit. The full characterization of the NE213 detector+digital system in terms of pulse height resolution parameters and light output function for neutrons will be the next step to be carried out in order to perform neutron spectrometry by means of unfolding codes.

\section{Acknowledgments}

The authors would like to thank Dr. H. Schuhmacher and Dr. H. Klein for their comments on the manuscript and the staff of the PTB neutron irradiation facility for the support in the experiments.

\section{References}

[1] Y. Kaschuck, B. Esposito, Nuclear Instruments and Methods in Phys. Res. A 551 (2005) 420

[2] M. Riva, B. Esposito, D. Marocco, Proceedings of $10^{\text {th }}$ ICALEPCS Int. Conf. on Accelerator \& Large Expt. Physics Control Systems. Geneva, 10-14 Oct 2005, P-O2.041-4, http://icalepcs2005.web.cern.ch/icalepcs2005/ (2005)

[3] B. Esposito et al., Review of Scientific Instruments, 75(10) (2004) 3550

[4] A. Zimbal et al., Review of Scientific Instruments, 75(10) (2004) 3553

[5] H. Klein, S. Neumann, Nuclear Instruments and Methods in Phys. Res. A 476 (2002) 132 